\title{
Physical conditions of ionized gas and stellar populations in circumnuclear starbursts
}

\author{
Johan H. Knapen ${ }^{1,2}$, Lisa M. Mazzuca ${ }^{3}$ and Marc Sarzi ${ }^{2}$ \\ ${ }^{1}$ Instituto de Astrofísica de Canarias, E-38200 La Laguna, Spain \\ ${ }^{2}$ University of Hertfordshire, Hatfield, AL10 9AB, UK \\ ${ }^{3}$ NASA Goddard Space Flight Center, Greenbelt, MD 20771, USA
}

\begin{abstract}
We present an emission-line diagnostic analysis of integral-field spectroscopic observations that cover the circumnuclear ring-like regions in a small number of spiral galaxies. We concentrate on the specific case of the Sa galaxy NGC 7742, which hosts a spectacular circumnuclear starburst ring and nuclear regions characterised by low-ionisation emission. The gas in the ring rotates in the opposite sense to the stars in the galaxy, suggesting a recent merging or acquisition event. The combination of integral-field measurements for the $\mathrm{H} \alpha+\mathrm{N}[\mathrm{II}]$ emission lines from DensePak and the $\mathrm{H} \beta$ and [OIII] emission from SAURON allows the construction of diagnostic diagrams that highlight the transition from star formation in the ring to excitation by high-velocity shocks or by an AGN near the centre. DensePak measurements for the [SII] line ratio reveal very low gas densities in the ring, $N_{\mathrm{e}}<100 \mathrm{~cm}^{-3}$, characteristic of massive HiI regions. Comparison with MAPPINGS III models for starbursts with low gas densities shows that the ring is of roughly solar metallicity. We suggest that NGC 7742 cannibalised a smaller galaxy rich in metal-poor gas, and that star formation episodes in the ring have since increased the metallicity to its present value.
\end{abstract}

Keywords. galaxies: evolution, galaxies: individual (NGC 7742), galaxies: ISM, galaxies: kinematics and dynamics, galaxies: nuclei, galaxies: spiral, galaxies: starburst

\section{Introduction}

We study the circumnuclear ring of massive star formation (SF) in the non-barred galaxy NGC 7742. Nuclear rings of massive SF occur in some $20 \%$ of all spiral galaxies, and mostly occur in barred galaxies (Knapen 2005). Understanding non-barred nuclear ring hosts is important to confirm our dynamical understanding of such rings (Knapen et al. 1995).

In the case of NGC 7742, the questions we set out to answer are how is the nuclear ring fuelled in the absence of a large-scale bar; and how can it co-exist with the counterrotating gas and stars in the central kpc region (as reported by de Zeeuw et al. 2002)?

In our study, we combined two integral-field datasets: SAURON in the blue (FalcónBarroso et al. 2006), and DENSEPAK in the red optical range (Mazzuca et al. 2006).

\section{Results}

From the [SII] line ratio, we can first estimate the electron density $N_{\mathrm{e}}$ to be low (Fig. 1, left): we assume $N_{\mathrm{e}}=10 \mathrm{~cm}^{-3}$ in our subsequent modelling (Mazzuca et al. 2006).

We then use diagnostic diagrams to compare blue and red emission line ratios (Fig. 1, right). Comparison with MAPPINGS III models (Kewley et al. 2001) shows that the emission in the nuclear ring is powered by SF; that there is little variation in the physical parameters of the gas around the ring; and that the metallicity is Solar. 

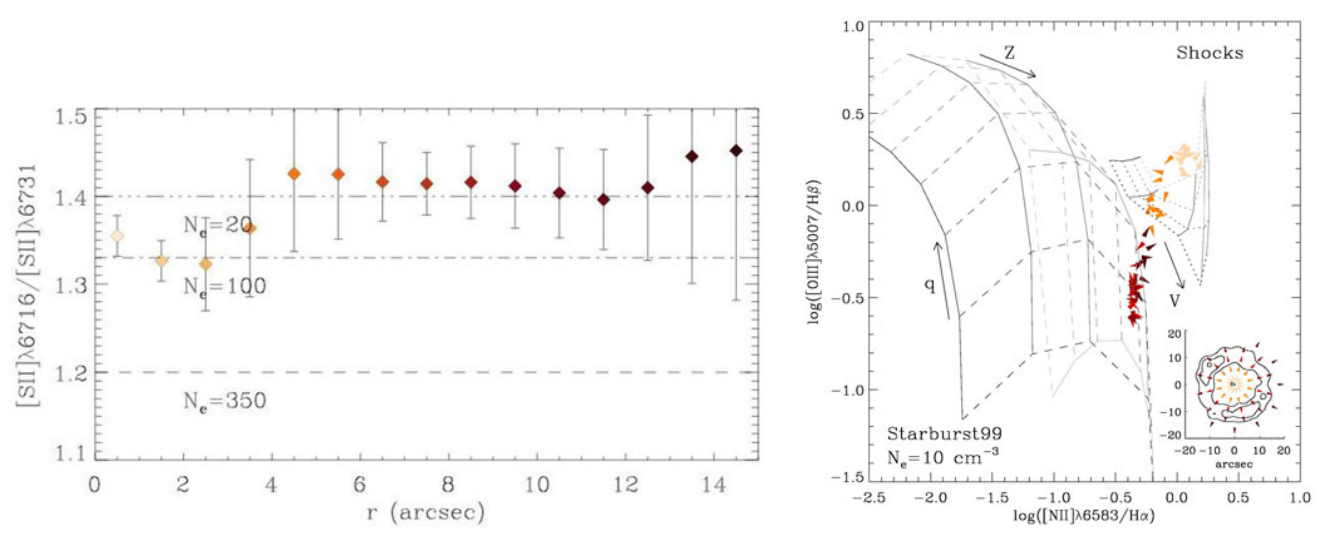

Figure 1. [SII] line ratio as a function of radius, yielding an estimate of the electron density (left) and the $[\mathrm{OIII}] / \mathrm{H} \beta$ vs. $[\mathrm{NII}] / \mathrm{H} \alpha$ diagnostic diagram (right) for the central region of NGC 7742 (from Mazzuca et al. 2006).

We interpret all this as follows:

- The counter-rotation is caused by the past merger of NGC 7742 with a small gas-rich galaxy.

- The gas in this small galaxy must have had low metallicity, but subsequent bursts of $\mathrm{SF}$ in the nuclear ring zone have enriched the metallicity of the gas to Solar (Allard et al. 2007; see also these proceedings, p. 000).

- The merger caused an asymmetry in the gravitational potential of NGC 7742, which in turn caused the inflow of gas and the subsequent formation of the nuclear ring.

\section{Conclusions}

We have pioneered an approach to combine two sets of integral-field observations of the central regions of galaxies, in blue and red optical, to complement stellar population studies with a comprehensive investigation of the physical properties of the emitting gas.

We confirm emission by SF in the rings, little azimuthal variation around the rings, and Solar metallicity.

The picture of NGC 7742 is of a past minor merger, followed by episodic SF in the ring region which increased the metallicity to the observed Solar value.

\section{References}

Allard, E. L., Sarzi, M., Knapen, J. H. \& Mazzuca, L. M. 2007, MNRAS, submitted Falcón-Barroso, J. \& the SAURON team 2006, MNRAS 369, 259

Kewley, L. J., Dopita, M. A., Sutherland, R. S., Heisler, C. A. \& Trevena, J. 2001, ApJ 556, 121

Knapen, J. H. 2005, A\&A 429, 141

Knapen, J. H., Beckman, J. E., Heller, C. H., Shlosman, I. \& de Jong, R. S. 1995, ApJ 454, 623

Mazzuca, L. M., Sarzi, M., Knapen, J. H., Veilleux, S. \& Swaters, R. 2006, ApJ 649, L77

de Zeeuw, T. \& the SAURON team 2002, MNRAS 329, 513 